\title{
Evaluation of the effects of antiarthritic drugs on the secretion of proteoglycans by lapine chondrocytes using a novel assay procedure
}

\author{
SIMON COLLIER AND PETER GHOSH
}

From the Raymond Purves Research Laboratories (The University of Sydney) at the Royal North Show Hospital of Sydney, St Leonards, NSW 2065, Australia

SUMmARY A new method is described for separating free ${ }^{35} \mathrm{SO}_{4}^{--}$from ${ }^{35} \mathrm{SO}_{4}$ labelle proteoglycans synthesised by rabbit articular chondrocytes cultured in the presence of excess ${ }^{35} \mathrm{SO}_{4}^{-}$. The procedure uses the low solubility product of barium sulphate to remove, by precipitation, free ${ }^{35} \mathrm{SO}_{4}^{--}$from culture medium. Optimum recovery of ${ }^{35} \mathrm{SO}_{4}$ labelled proteoglycans was achieved after papain digestion to release ${ }^{35} \mathrm{SO}_{4}$-glycosaminoglycans, an addition of chondroitin sulphate before the precipitation step. Using this assay, we studied the

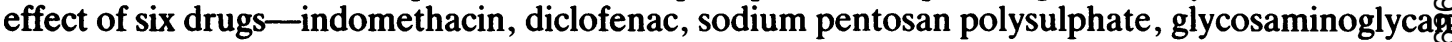
polysulphate ester, tiaprofenic acid, and ketoprofen-on the secretion into the medium of labelled proteoglycans by lapine chondrocytes. The six drugs were tested at $0 \cdot 1,1,10,50$, and 100 $\mu \mathrm{g} / \mathrm{ml}$ over four consecutive 48 hour culture periods. A consistent concentration-response patter was found for the four non-steroidal anti-inflammatory drugs (NSAIDs) studied. Generally they inhibited proteoglycan secretion at 50 and $100 \mu \mathrm{g} / \mathrm{ml}$ but had no effect at lower concentration $\$$ Inhibition of secretion was strongest with indomethacin and diclofenac at 50 and $100 \mu \mathrm{g} / \mathrm{ml}$. I contrast with the NSAIDs studied, the two sulphated polysaccharides (sodium pentosa polysulphate and glycosaminoglycan polysulphate ester) at low concentrations increased proteoglycan secretion by chondrocytes, with maximal stimulation occurring at $1 \mu \mathrm{g} / \mathrm{ml}$. Sodium pentosan polysulphate, but not glycosaminoglycan polysulphate ester, showed inhibitory activity at 50 and $100 \mu \mathrm{g} / \mathrm{ml}$.

Key words: non-steroidal anti-inflammatory drugs, sodium pentosan polysulphate, glycosaminis glycan polysulphate ester, diclofenac, indomethacin, ketoprofen, tiaprofenic acid.

Non-steroidal anti-inflammatory drugs (NSAIDs) still represent the mainstay of therapeutic management of osteoarthritis. It is now recognised, however, that such drugs, although effective at relieving the symptoms of the disease, may do little to improve the condition, and in some instances may contribute to its progression (see review by Burkhardt and Ghosh $^{\mathrm{f}}$ ). In recent years new NSAIDs and other drugs have become commercially available which are reported to be free of deleterious effects on

Accepted for publication 18 October 1988.

Correspondence to Associate Professor Peter Ghosh, Raymond Purves Research Laboratories (The University of Sydney) at the Royal North Shore Hospital of Sydney, St Leonards, NSW 2065, Australia. cartilage metabolism, but it is often difficult to assess the validity of such claims because of the limitations of the methods used. Ideally the influence of a drug on cartilage cells should be monitored of different drug concentrations, over different time periods, and under carefully controlled condition but in many cases these criteria are not full\$ observed. The most convenient means of assessing the effects of drugs on chondrocytes is by in vitro assays. Although this approach has its shortcomings it can provide a primary screening system, which cas be followed up by animal experiments. Measurement of proteoglycan production by cartilage explants of chondrocyte monolayers generally involves pulsing the cultures with ${ }^{35} \mathrm{SO}_{4}^{--}$to radiolabel the newt 
synthesised proteoglycans. This method, however, requires the subsequent separation of the resulting ${ }^{35} \mathrm{SO}_{4}$ labelled proteoglycans from the large amounts of unincorporated ${ }^{35} \mathrm{SO}_{4}^{--}$. This may be achieved by removal of the free ${ }^{35} \mathrm{SO}_{4}^{--}$by gel filtration chromatography of each sample, ${ }^{2}$ exhaustive dialysis of individual samples, ${ }^{3}$ or papain digestion and cetylpyridium chloride precipitation of the ${ }^{35} \mathrm{SO}_{4}$-glycosaminoglycans. ${ }^{4}$ These methods are time consuming and tedious and, in the latter method in particular, the number of steps involved could result in significant cumulative experimental errors.

In the present study we describe a rapid, simple, and reproducible method for separating free ${ }^{35} \mathrm{SO}_{4}^{--}$ from ${ }^{35} \mathrm{SO}_{4}$ labelled proteoglycans in chondrocyte or cartilage cultures. The efficiency of this method allows for comprehensive comparative experiments of the effects of drugs on the production of proteoglycans by chondrocytes under a variety of conditions. We used this method to compare the effects of six currently available antiarthritic agents, each at five concentrations, on the total proteoglycan secretion of cultured rabbit articular chondrocytes, measured for four consecutive 48 hour culture intervals.

\section{Materials and methods}

A N IM A L S

New Zealand white rabbits (6-8 weeks old) of either sex were obtained from the University of Sydney animal house, Castle Hill, NSW, Australia.

\section{PREPARATION OF PRIMARY CHONDROCYTE} C ULTURES

Rabbits were killed by intracardiac injection of pentobarbitone (May and Baker Australia, Pty Ltd, West Footscray, Victoria, Australia). Both hind legs were removed, and, under aseptic conditions, the knee joints were opened and the articular cartilage was shaved off the femoral condyles, the tibial plateaux, and the patellae. The shavings were transferred to a $15 \mathrm{ml}$ plastic centrifuge tube (Corning Laboratory Science Company, Stone, Staffordshire, UK) containing phosphate buffered saline (PBS). After washing with two $15 \mathrm{ml}$ volumes of PBS the fragments were resuspended in $15 \mathrm{ml}$ of $0.25 \%$ trypsin (1:250, Gibco Laboratories, Grand Island, New York, USA) in PBS and incubated at $37^{\circ} \mathrm{C}$ for one hour. The trypsin solution was aspirated and replaced with $15 \mathrm{ml}$ of a solution of $0.2 \%$ collagenase (type 1A, Sigma Chemical Company, St Louis, MO, USA) in Ham's F12 medium (Flow Laboratories, McLean, Va, USA) containing $10 \%$ fetal calf serum (FCS) (Commonwealth Serum Laboratories, Parkville, Victoria, Australia),
$80 \mathrm{U} / \mathrm{ml}$ gentamicin (David Bull Laboratories Pty Ltd, Rydalmere, NSW, Australia), 20 mM HEPES ( $N$-2-hydroxyethylpiperazine- $N^{\prime}$-2-ethanesulphonic acid; Sigma Chemical Company, St Louis, MO, USA), and incubation was continued at $37^{\circ} \mathrm{C}$ for three hours with occasional shaking. The digest was washed three times with $15 \mathrm{ml}$ volumes of Ham's F12 medium plus $10 \%$ FCS (F12-FCS), the cells were counted with a haemocytometer, and $2 \times 10^{6}$ viable cells in $12 \mathrm{ml} \mathrm{F12-FCS} \mathrm{were} \mathrm{plated} \mathrm{into} 75 \mathrm{~cm}^{2}$ tissue culture flasks (Miles Laboratories, Naperville, IL, USA). A preparation from one rabbit typically yielded approximately $10 \times 10^{6}$ chondrocytes. The medium was changed after three days and subsequently every two to three days. Confluence was reached by the chondrocyte cultures in six to eight days.

\section{PREPARATION OF TEST CULTURES}

Six drugs were tested for their effects on proteoglycan secretion by articular chondrocyte cultures. They were glycosaminoglycan polysulphate ester (Luitpold Werk, Munich, FRG), sodium pentosan polysulphate (Benechemie, Munich, FRG), indomethacin (Merck Sharp and Dohme Australia Pty Ltd, Granville, NSW, Australia), diclofenac sodium (Ciba Geigy Australia Ltd, Pendle Hill, NSW, Australia), ketoprofen (May and Baker Australia Pty Ltd, West Footscray, Victoria, Australia), and tiaprofenic acid (Roussel Pharmaceuticals Pty Ltd, Castle Hill, NSW, Australia).

Medium was decanted from confluent chondrocyte cultures, cells were washed with two $5 \mathrm{ml}$ volumes of PBS, and then $3 \mathrm{ml}$ of $0.2 \%$ trypsin, $0.1 \%$ edetic acid (Ajax Chemical Company, Sydney, Australia) in PBS was added, and cultures were incubated at $37^{\circ} \mathrm{C}$ for two to three minutes until the cells detached. The cells were aspirated and washed in three $15 \mathrm{ml}$ volumes of F12-FCS, then counted, and the concentration adjusted to $2 \times 10^{5}$ cells $/ \mathrm{ml}$. The cell suspension was dispensed into the wells $\left(10^{5}\right.$ cells/well in $0.5 \mathrm{ml}$ ) of 24-well plates (Flow Laboratories, McLean, Va, USA). At this plating density the cells had attached to form confluent monolayers after an overnight incubation. The medium was changed and each well received $450 \mu \mathrm{l}$ of F12FCS containing $4.3 \times 10^{6}$ disintegrations per minute ${ }^{35} \mathrm{SO}_{4}^{--}(92.5 \mathrm{kBq} /$ well) (New England Nuclear Corp, Bedford, MA, USA). The various drugs were dissolved and appropriately diluted in serum free Ham's F12 medium and delivered to the wells in $50 \mu$ l volumes, to give final volumes in the wells of $500 \mu \mathrm{l}$ and the desired final drug concentrations of 0 , $0 \cdot 1,1,10,50$, and $100 \mu \mathrm{g} / \mathrm{ml}$. Four replicate wells were used for each drug concentration. Sufficient F12-FCS plus ${ }^{35} \mathrm{SO}_{4}^{--}$was prepared in a single batch 
for the experiment to ensure that all wells were exposed to an equal concentration of ${ }^{35} \mathrm{SO}_{4}^{--}$at each medium change. Medium was changed and fresh drug solutions were added after two, four, and six days of culture. At these medium changes and on day 8 when the experiment was terminated the exhausted medium was pipetted from each well into tubes and stored for assay.

${ }^{35} \mathrm{SO}_{4}$ - PROTEOGLYCAN ASSAY

The level of secretion of labelled proteoglycans by the chondrocytes was measured by separating free (unincorporated) ${ }^{35} \mathrm{SO}_{4}^{--}$from ${ }^{35} \mathrm{SO}_{4}$ labelled proteoglycans, as described below. The principle of the separation method is based on the insolubility of barium sulphate $\left(\mathrm{BaSO}_{4}\right)$. The chondrocyte culture supernatants $(500 \mu \mathrm{l})$, which were collected into $75 \times 10 \mathrm{~mm}$ plastic tubes (Edwards Medical Supplies, Narellan, NSW, Australia), were subjected to papain digestion to release glycosaminoglycans. The stock solution used was $2.5 \mathrm{mg} / \mathrm{ml}$ papain (crude powder) (Sigma Chemical Co, MO, USA), 7.9 $\mathrm{mg} / \mathrm{ml}$ cysteine- $\mathrm{HCl}$ (Sigma Chemical Co, MO, USA) in PBS preactivated at $60^{\circ} \mathrm{C}$ for 30 minutes. Papain stock solution $(125 \mu \mathrm{l})$ was added to each tube, which was capped and incubated at $60^{\circ} \mathrm{C}$ for two hours. After papain digestion $300 \mu$ l of solution containing equal volumes of $0.2 \mathrm{M} \mathrm{Na}_{2} \mathrm{SO}_{4}$ and $50 \mathrm{mg} / \mathrm{ml}$ chondroitin sulphate (crude shark and whale, Sigma Chemical Co, MO, USA) was added to each tube. Tubes were vortexed, $0.4 \mathrm{M} \mathrm{BaCl}_{2}$ $(150 \mu \mathrm{l})$ was then added, tubes were again vortexed and centrifuged for 10 minutes at $1500-2000 \mathrm{~g}$. The precipitation step was then repeated on the supernatant. From each tube $500 \mu \mathrm{l}$ of supernatant was pipetted and transferred to a clean tube, great care being taken not to disturb the precipitate. One hundred microlitres of a 2:5 solution of chondroitin sulphate $(50 \mathrm{mg} / \mathrm{ml})$ and $\mathrm{BaCl}_{2}(0.4 \mathrm{M})$ was added, tubes were vortexed, $100 \mu \mathrm{l}$ of $0.2 \mathrm{M} \mathrm{Na}_{2} \mathrm{SO}_{4}$ was added, tubes were vortexed again, and centrifuged as before. Aliquots $(100 \mu \mathrm{l})$ of this supernatant were carefully removed by pipette (again great care being taken not to disturb the precipitate) and transferred to scintillation vials (Packard Instruments Co Inc, Downers Grove, IL, USA). The radioactivity in the samples was determined by routine $\beta$ scintillation counting. As only relative proteoglycan synthesis under different culture treatments was of interest the results were expressed as disintegrations per minute of ${ }^{35} \mathrm{SO}_{4}$ labelled proteoglycan $/ 10^{5}$ cells.

PREPARATION OF PURIFIED

${ }^{35} \mathrm{SO}_{4}$ - PROTEOGL Y CAN

Confluent rabbit chondrocyte cultures in $75 \mathrm{~cm}^{2}$ flasks containing $12 \mathrm{ml}$ of medium were pulsed with
${ }^{35} \mathrm{SO}_{4}^{--}(185 \mathrm{kBq} / \mathrm{ml})$ for 48 hours. The medium was removed and concentrated to approximately $5 \mathrm{~m}$ using an Amicon YM10 membrane (Amicon Corp Danvers, MA, USA). The sample was then applied to a $1.6 \times 90 \mathrm{~cm}$ Sepharose CL2B column (Pharmaci㽚 South Seas Pty Ltd, Sydney, NSW, Australia) which had been precalibrated with DNA $\left(\mathrm{V}_{\mathrm{o}}\right)$ and $\mathrm{Na}_{2}{ }^{35} \mathrm{SO}_{4}\left(\mathrm{~V}_{t}\right)$. The column was eluted with $0.5 \mathrm{No}$ sodium acetate $(\mathrm{pH} \mathrm{6.8)}$ at a flow of $10 \mathrm{ml} / \mathrm{h}$ and fractions $(3.0 \mathrm{ml})$ monitored for radioactivity. The ${ }^{35} \mathrm{SO}_{4}$-proteoglycans eluted at $\mathrm{V}_{\mathrm{o}}$ as a single peak of aggregated proteoglycans and free ${ }^{35} \mathrm{SO}_{4}^{--}$eluted as $\mathrm{V}_{\mathrm{t}}$. The $\mathrm{V}_{\mathrm{o}}$ fractions were pooled and used as $\underset{\mathbb{Q}}{\mathrm{Q}}$ source of purified ${ }^{35} \mathrm{SO}_{4}$-proteoglycans to validate the proteoglycan assay. The specific activity of ther ${ }^{35} \mathrm{SO}_{4}$-proteoglycan preparation determined after hexuronate analysis ${ }^{5}$ was $15000 \mathrm{cpm} / \mu \mathrm{g}$ hexuroni acid.

STATISTICAL ANALYSIS

The data generated on the effects of each drug studied on proteoglycan synthesis were analysed by two factor analysis of variance. Each analysis had six rows (drug concentrations: $0,0 \cdot 1,1,10,50$, and 1000 $\mu \mathrm{g} / \mathrm{ml}$ ) and four columns (culture periods: day $0-2$, 2-4, 4-6, and 6-8) and tested three null hypotheses. (1) that there was no significant difference in the proteoglycan synthesis of cultures exposed to any ofD the six drug concentrations (difference between rows); (2) that there was no significant difference ing the proteoglycan synthesis of cultures during anys of the four culture periods (difference between columns); and (3) that the 'dose-reponse' to the drug was not significantly different at any of the fouro culture periods (interaction). As all the null hypotheses proposed were rejected and the differences were highly significant (see Figs 1-6), the significanto differences between all 24 means $(6 \times 4)$ were determined using the Student-Neuman-Keules tes $\varrho$ and the $5 \%$ level of the $Q$ distribution. ${ }^{6}$

\section{Results}

PROTEOGLY CA N A S A Y
To separate free ${ }^{35} \mathrm{SO}_{4}^{--}$from ${ }^{35} \mathrm{SO}_{4}$-proteoglycan in the chondrocyte culture supernatant we used the very low solubility product of $\mathrm{BaSO}_{4}\left(1.2 \times 10^{-1} \phi\right.$ relative to the moderate solubility of the glycos aminoglycan barium salts. Table 1 summarises the development of the method, in which free ${ }^{35} \mathrm{SO}_{4}^{-}{ }^{-} \mathrm{O}$ and purified ${ }^{35} \mathrm{SO}_{4}$-proteoglycan and ${ }^{35} \mathrm{SO}_{4}$-glycoso aminoglycans were subjected to $\mathrm{BaSO}_{4}$ precipitatior to arrive at conditions for optimum recovery. It car be seen from Table 1 that in an experimentab situation with pulsed chondrocyte culture super natant the addition of excess $\mathrm{Ba}^{++}$to samples woul\& 
Table 1 Optimisation of the separation of free ${ }^{35} \mathrm{SO}_{4}^{--}$from ${ }^{35} \mathrm{SO}_{4}$-proteoglycans using barium sulphate precipitation

Disintegrations per minute (dpm) in $100 \mu$ l supernatant

After 1st precipitation After 2nd precipitation

and centrifugation and centrifugation

$\overline{d p m} \quad$ Recovery $(\%) \quad$ Recovery $(\%)$

Free $^{35} \mathrm{SO}_{4}^{--}$

Not precipitated with $\mathrm{BaSO}_{4}$

$\mathrm{BaSO}_{4}$ precipitated minus chondroitin sulphate

$\mathrm{BaSO}_{4}$ precipitated plus chondroitin sulphate

Non-digested ${ }^{35} \mathrm{SO}_{4}$-proteoglycan

Not precipitated with $\mathrm{BaSO}_{4}$

$\mathrm{BaSO}_{4}$ precipitated minus chondroitin sulphate

$\mathrm{BaSO}_{4}$ precipitated plus chondroitin sulphate

Papain digested ${ }^{35} \mathrm{SO}_{4}$ proteoglycan

Not precipitated with $\mathrm{BaSO}_{4}$

$\mathrm{BaSO}_{4}$ precipitated minus chondroitin sulphate

$\mathrm{BaSO}_{4}$ precipitated plus chondroitin sulphate

$\begin{array}{rr}543171 & - \\ 207 & \\ 1603 & \end{array}$

- 0

0

387980
34
126

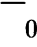

0

$\begin{array}{rrr}1 & 407 & - \\ 1 & 50 & 4 \\ 1 & 227 & 87\end{array}$

4
87

1005

23

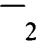

2

1330
593
1363

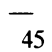

102

Free ${ }^{35} \mathrm{SO}_{4}^{--}$alone, ${ }^{35} \mathrm{SO}_{4}$-proteoglycan, and papain digested ${ }^{35} \mathrm{SO}_{4}$-proteoglycan were subjected to two successive precipitations with excess $\mathrm{Ba}^{++}$and carrier chondroitin sulphate (see text). In each case precipitations were carried out with and without exogenous chondroitin sulphate. The data demonstrate how the precipitation method removes free ${ }^{35} \mathrm{SO}_{4}$ from the ${ }^{35} \mathrm{SO}_{4}$-proteoglycan but that (i) exogenous chondroitin sulphate is required to prevent precipitation of ${ }^{35} \mathrm{SO}_{4}$-proteoglycan; (ii) the presence of this chondroitin sulphate increases free ${ }^{35} \mathrm{SO}_{4}^{--}$background, and so requires a second precipitation to reduce free ${ }^{35} \mathrm{SO}_{4}^{--}$to negligible levels. Even in the presence of chondroitin sulphate there was some precipitation of ${ }^{35} \mathrm{SO}_{4}$-proteoglycan unless it was previously digested to ${ }^{35} \mathrm{SO}_{4}$-glycosaminoglycans with papain.

not be sufficient, for although $\mathrm{Ba}^{35} \mathrm{SO}_{4}$ would be precipitated, so too would almost all of the labelled proteoglycans $(4 \%$ recovery in Table 1$)$. Digestion of the proteoglycan aggregates with papain to yield free ${ }^{35} \mathrm{SO}_{4}$-glycosaminoglycan reduced the loss upon precipitation to $55 \%$. Addition of exogenous chondroitin sulphate to the ${ }^{35} \mathrm{SO}_{4}$-glycosaminoglycans before precipitation, however, afforded $100 \%$ recovery of ${ }^{35} \mathrm{SO}_{4}$-glycosaminoglycans in the supernatant. When large amounts of ${ }^{35} \mathrm{SO}_{4}^{--}$were present in supernatants the background radioactive level after a single precipitation step was still relatively high, but after a second precipitation step the total recovery of ${ }^{35} \mathrm{SO}_{4}$-glycosaminoglycans was preserved while the ${ }^{35} \mathrm{SO}_{4}^{--}$background was reduced more than 10-fold to an acceptably low level.

\section{EFFECT OF ANTIARTHRITIC DRUGS ON PROTEOGLYCAN BIOSYNTHESIS BY RABBIT CHONDROCYTES}

Chondrocyte cultures were exposed to drugs and ${ }^{35} \mathrm{SO}_{4}^{--}$for four consecutive 48 hour culture intervals, and the ${ }^{35} \mathrm{SO}_{4}$-proteoglycans which had accumulated in the medium at the end of each culture interval were determined by the papain/ double precipitation procedure described above. The six drugs, glycosaminoglycan polysulphate ester, sodium pentosan polysulphate, indomethacin, diclofenac, ketoprofen, and tiaprofenic acid, were tested at concentrations of 0 (control), $0 \cdot 1,1,10,50$, and
$100 \mu \mathrm{g} / \mathrm{ml}$. Figures $1-6$ show the results obtained for each drug. As the purpose of this study was to compare the effects of drugs at different concentrations relative to a drug free control culture the data presented represent the disintegrations per minute of one fifteenth (dilution due to the assay) of the ${ }^{35} \mathrm{SO}_{4}$-glycosaminoglycans produced by $10^{5}$ cells during the indicated 48 hour periods. For all cultures studied a common pattern of chondrocyte secretory activity was observed. During the first 48 hour period proteoglycan secretion was low, increasing to a maximum on days $2-4$. A progressive decline in activity then occurred over the next two culture intervals in all experiments (see Figs 1-6).

\section{Glycosaminoglycan polysulphate ester}

Figure 1 shows secretion of ${ }^{35} \mathrm{SO}_{4}$-proteoglycan by chondrocytes cultured in the absence and presence of increasing concentrations of glycosaminoglycan polysulphate ester over an eight day culture period. For the period days $0-2$ glycosaminoglycan polysulphate ester caused no significant effect on proteoglycan secretion up to a concentration of $10 \mu \mathrm{g} / \mathrm{ml}$, but at 50 and $100 \mu \mathrm{g} / \mathrm{ml}$ the drug slightly depressed proteoglycan secretion. During the next two day culture period the pattern of response to increasing concentrations of glycosaminoglycan polysulphate ester had changed substantially and became constant for the duration of the experiment. Inhibition of proteoglycan secretion was no longer observed at 


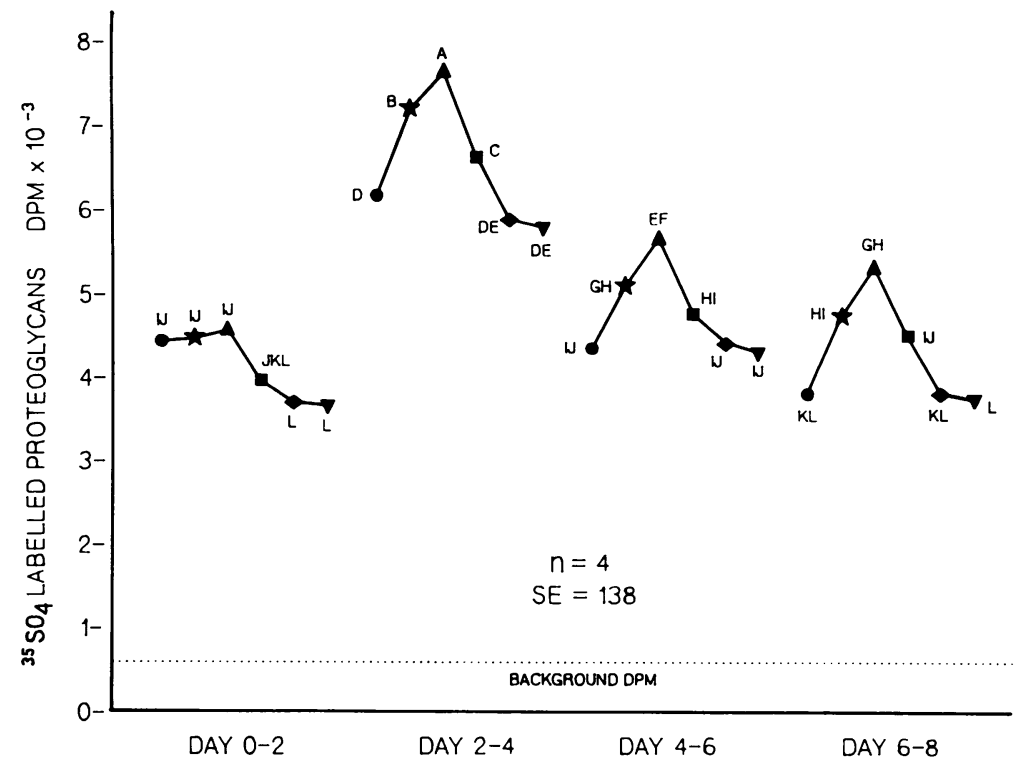

Fig. 1 Effect of glycosaminoglycan polysulphate ester on the secretion of proteoglycans by chondrocytes. Confluent chondrocyte monolayer cultures were grown for eight days, constantly exposed to the indicated drugs plus ${ }^{35} \mathrm{SO}_{4}^{--}$. The medium, containing fresh drugs and radiolabel, was changed every 48 hours and the exhausted medium from each well was retained for assay of the ${ }^{35} \mathrm{SO}_{4}$ proteoglycans which had accumulated during the previous 48 hours of culture. Each plotted curve comprising six data points is a concentration-response curve, the six concentrations being in every case, from left to right, $\bigcirc 0, \star 0 \cdot 1, \triangle 1 \cdot 0, \square 10, \diamond 50$, and $\nabla 100 \mu \mathrm{g} / \mathrm{ml}$. All values within each individual figure were compared by a single statistical analysis (see 'Materials and methods'), and the rankings of the magnitudes of the individual values (means of four culture wells) are indicated by the letters assigned to each point. Those means which do not share a letter in common are significantly different at the $5 \%$ level. Any two or more means which have a letter in common are not significantly different. For example, a value designated 'IJ' is not different from a value labelled ' $H I$ ' (both have the letter ' $I$ ') or from a value labelled ' $J K L$ ' (both have the letter ' $J$ '), but the value labelled ' $H I$ ' is significantly greater than the value labelled ' $J K L$ ' (no letter in common).

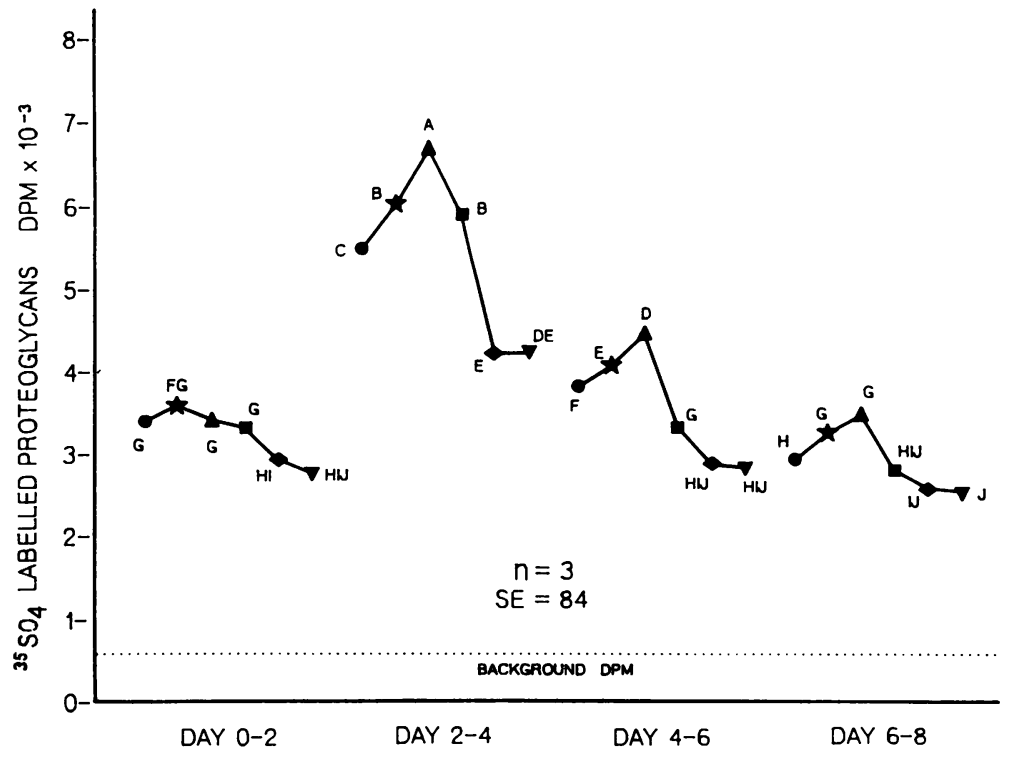

Fig. 2 Effect of sodium pentosan polysulphate at various concentrations on proteoglycan secretion by lapine chondrocytes. See legend to Fig. 1. 
any concentration, even at 50 and $100 \mu \mathrm{g} / \mathrm{ml}$ inhibition by glycosaminoglycan polysulphate ester was statistically indistinguishable from that of the controls. Glycosaminoglycan polysulphate ester at $0 \cdot 1,1$, and $10 \mu \mathrm{g} / \mathrm{ml}$, however, stimulted proteoglycan secretion significantly (except for days $4-6$ where $10 \mu \mathrm{g} / \mathrm{ml}$ was not significantly different from the control). Stimulation of proteoglycan secretion was maximal at $1 \mu \mathrm{g} / \mathrm{ml}$ over the two to four day culture period.
Sodium pentosan polysulphate

The response of chondrocytes to sodium pentosan polysulphate showed similarities with their response to glycosaminoglycan polysulphate ester (Fig. 2). A pattern was established by days 2-4, which persisted for the subsequent culture intervals. At days $0-2$ the proteoglycan secretion of the control chondrocytes and those cultured with $0 \cdot 1,1$, and $10 \mu \mathrm{g} / \mathrm{ml}$ sodium pentosan polysulphate were not significantly different. At 50 and $100 \mu \mathrm{g} / \mathrm{ml}$ of the
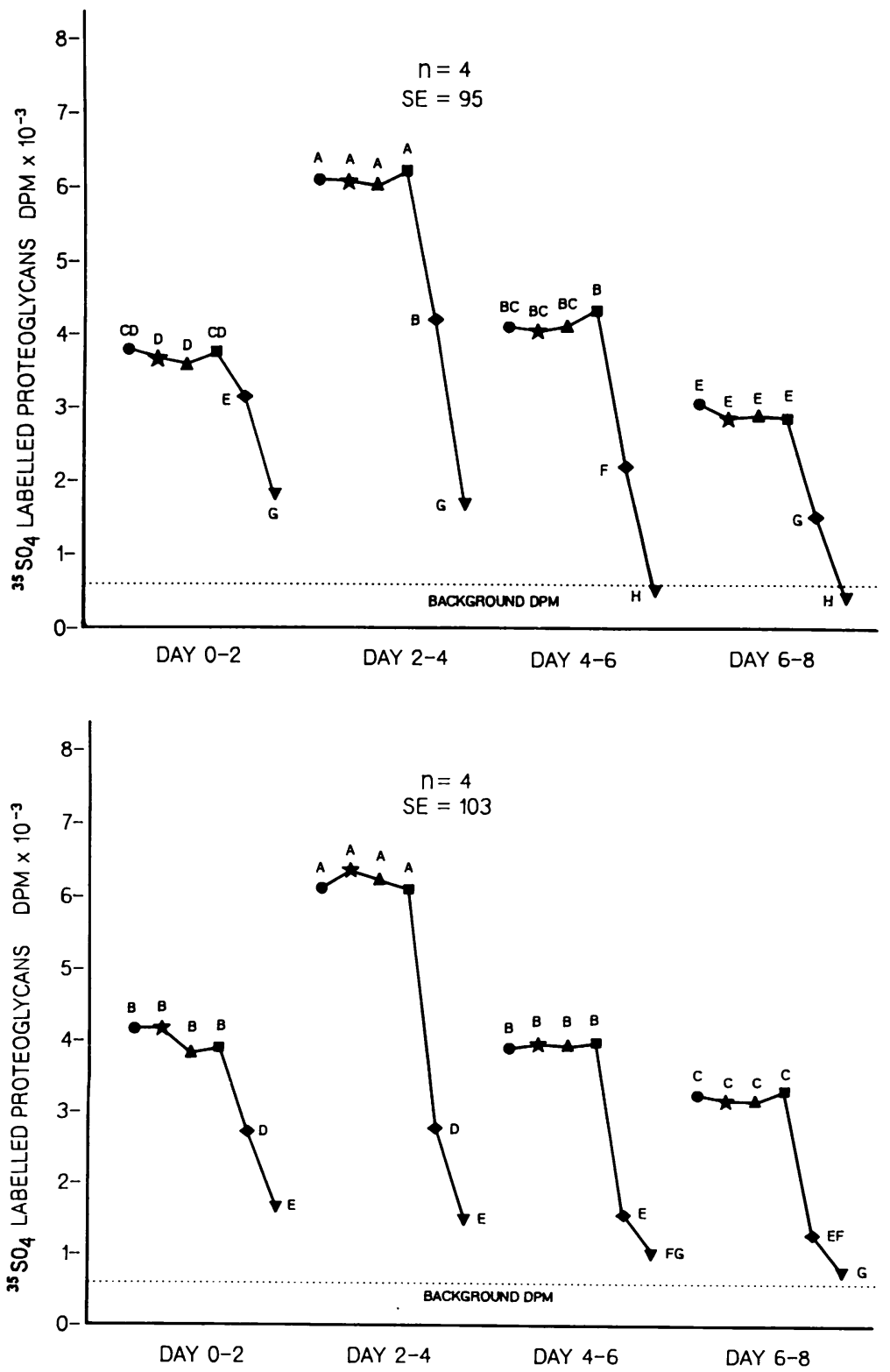

Fig. 3 Effect of indomethacin at various concentrations on proteoglycan secretion by lapine chondrocytes. See legend to Fig. 1.

Fig. 4 Effect of diclofenac at various concentrations on proteoglycan secretion by lapine chondrocytes. See legend to Fig. 1. 
drug proteoglycan secretion was inhibited. This significant diminution of proteoglycan secretion at high concentrations of sodium pentosan polysulphate, unlike glycosaminoglycan polysulphate ester, persisted throughout the experiment. Like glycosaminoglycan polysulphate ester, sodium pentosan polysulphate at $0 \cdot 1$ and $1 \mu \mathrm{g} / \mathrm{ml}$ stimulated proteoglycan secretion from day 2 onwards, the maximum stimulation being produced by $1 \mu \mathrm{g} / \mathrm{ml}$ at each two day culture interval. The response with time to $10 \mu \mathrm{g} / \mathrm{ml}$ sodium pentosan polysulphate was variable, the effect being stimulatory during days $2-4$, inhibitory during days $4-6$, and the same as that of the controls on days $0-2$ and days $6-8$.

\section{Indomethacin}

The pattern of response of the chondrocyte cultures to indomethacin was consistent over each culture interval (Fig. 3). Proteoglycan secretion was unaffected by concentrations of this drug of $0 \cdot 1,1$, and $10 \mu \mathrm{g} / \mathrm{ml}$, but was profoundly inhibited at 50 and $100 \mu \mathrm{g} / \mathrm{ml}$. By days $4-6{ }^{35} \mathrm{SO}_{4}$-proteoglycan secretion in the presence of $100 \mu \mathrm{g} / \mathrm{ml}$ indomethacin was essentially abolished, being reduced to the level of background disintegrations per minute.

\section{Diclofenac}

The pattern of proteoglycan secretory activity of chondrocytes cultured in medium containing diclofenac was found to be almost identical to that of cultures exposed to indomethacin (Fig. 4). Diclofenac at $0 \cdot 1,1$, and $10 \mu \mathrm{g} / \mathrm{ml}$ did not significantly influence proteoglycan secretion by chondrocytes. At 50 and $100 \mu \mathrm{g} / \mathrm{ml}$ of the drug, however, a major $\overrightarrow{\vec{F}}$ inhibitory effect on proteoglycan secretion was observed, with proteoglycan levels approaching $\frac{\bar{\sigma}}{-}$ background in the presence of $100 \mu \mathrm{g} / \mathrm{ml}$ of the drug $\overline{\bar{\omega}}$ during days $4-6$ and $6-8$. A comparison of Figs 3 and $\overrightarrow{\mathbb{Q}}$ 4 suggests that diclofenac at $50 \mu \mathrm{g} / \mathrm{ml}$ was slightly more inhibitory than indomethacin at $50 \mu \mathrm{g} / \mathrm{ml}$.

\section{Ketoprofen}

This drug elicited very little effect on proteoglycan $\vec{\sigma}$ secretion by rabbit chondrocytes over the concentra-@ tion range studied (Fig. 5). During days $0-2$ all? cultures secreted the same amount of ${ }^{35} \mathrm{SO}_{4}$-proteo- $-\infty$ glycan. The same was observed during days 6-8. Oner days 4-6 chondrocytes cultured in the presence of $100 \mu \mathrm{g} / \mathrm{ml}$ ketoprofen secreted significantly less ${ }_{\circ}^{N}$ proteoglycan than controls, but the magnitude of ${ }^{-}$ the reduction was modest. On days $2-4$, when $\vec{z}$ secretion levels were highest overall, a statistically@ significant diminution of proteoglycan secretion relative to control values resulted from both 50 and ${ }_{\infty}$ $100 \mu \mathrm{g} / \mathrm{ml}$ ketoprofen, but the diminished levels of ${ }^{\oplus}$ proteoglycan secretion resulting from these two drug concentrations were not distinguishable statistically from each other.

\section{Tiaprofenic acid}

The proteoglycan secretion of articular chondrocytes $\overrightarrow{\vec{A}}$ cultured in the presence of tiaprofenic acid was 3 broadly similar to that observed with ketoprofen, but concentration effects were more pronouncedo

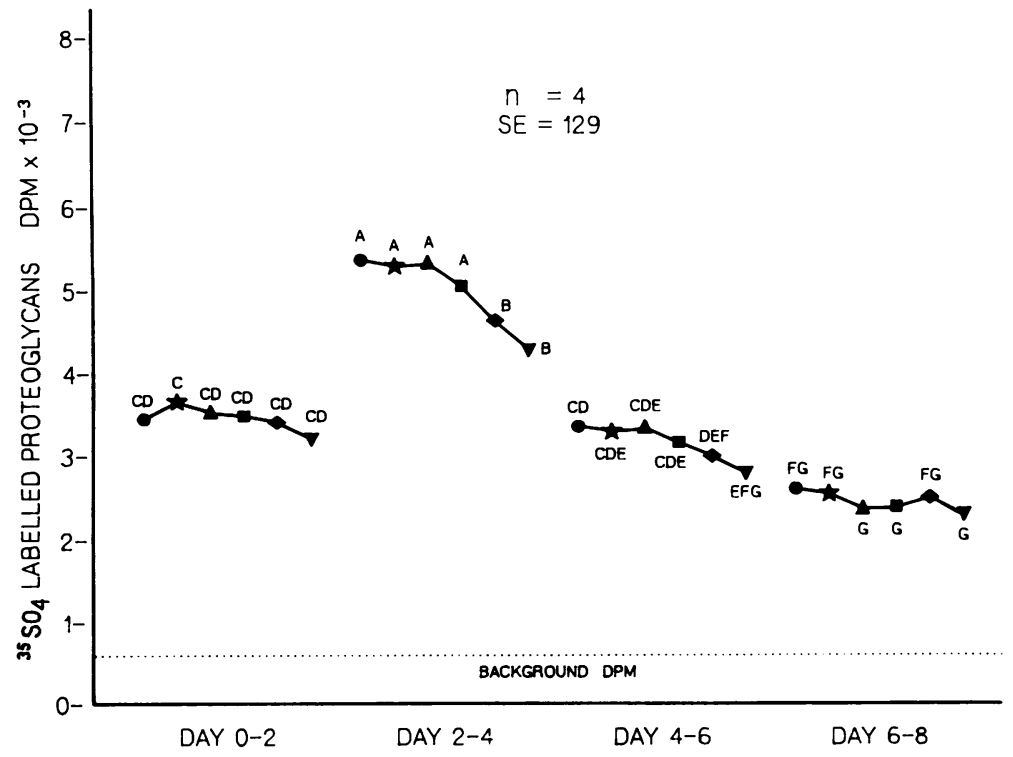

Fig. 5 Effect of ketoprofen at various concentrations on proteoglycan secretion by lapine chondrocytes. See legend to Fig. 1. 


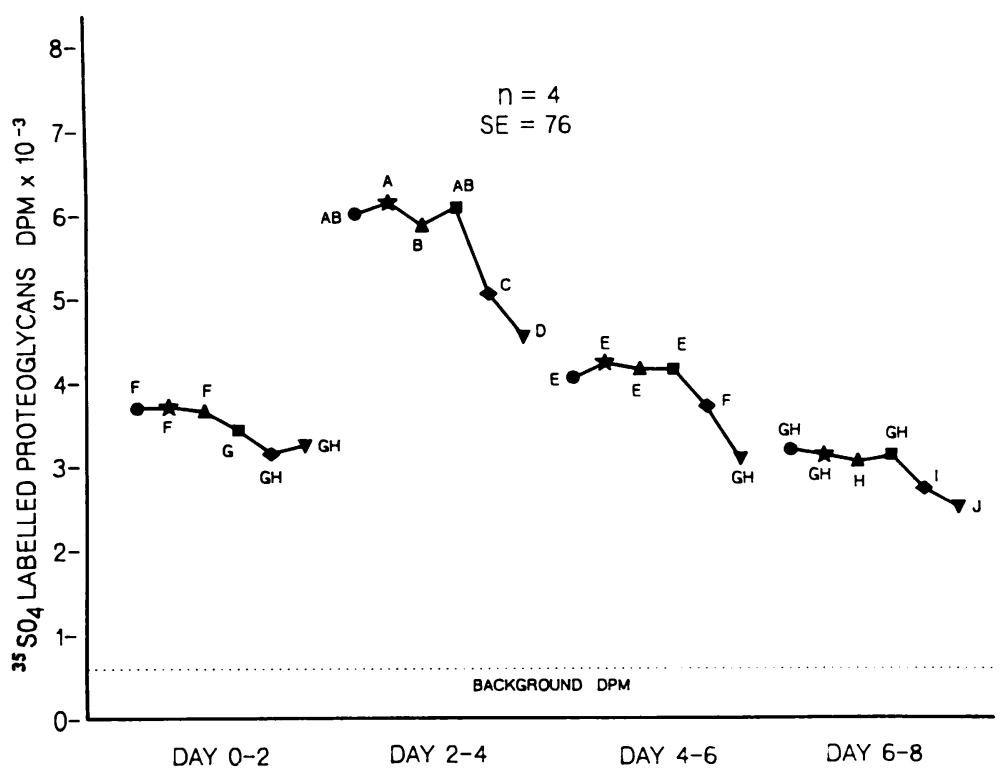

Fig. 6 Effect of tiaprofenic acid at various concentrations on proteoglycan secretion by lapine chondrocytes. See legend to Fig. 1.

(Fig. 6). During days 0-2 there was a slight but significant reduction in proteoglycan secretion at concentrations of 10,50 , and $100 \mu \mathrm{g} / \mathrm{ml}$, but the proteoglycan levels produced at these three drug concentrations were not different from one another. During the two to four day culture period the drug at $0 \cdot 1,1$, and $10 \mu \mathrm{g} / \mathrm{ml}$ had no significant effect on secretion compared with the controls, whereas $50 \mu \mathrm{g} / \mathrm{ml}$ and $100 \mu \mathrm{g} / \mathrm{ml}$ tiaprofenic acid reduced proteoglycan secretion. This pattern was repeated in the following culture period (days 4-6) and maintained for days $6-8$. In this last culture period, however, the magnitude of the reduction in the level of proteoglycan secretion at 50 and $100 \mu \mathrm{g} / \mathrm{ml}$ tiaprofenic acid, though still significant, was only slight.

\section{Discussion}

Our objective in the present study was to devise a simple in vitro model which would permit a thorough comparison of drug effects over a range of concentrations and time periods. Of particular importance was the need to maintain reproducibility by the elimination of time consuming steps, such as multicolumn chromatographic separations or techniques involving overnight precipitation and subsequent washing steps, which would introduce cumulative losses. As is evident from Table 1 the use of the low solubility of $\mathrm{BaSO}_{4}$ in conjunction with papain digestion and the introduction of chondroitin sulphate as a carrier permitted a satisfactory separation of unincorporated ${ }^{35} \mathrm{SO}_{4}^{--}$from ${ }^{35} \mathrm{SO}_{4}$ labelled glycosaminoglycans released by cells into the culture medium.

From the results it was evident that the six drugs studied fell into three groups of two, which had similar effects on proteoglycan secretion by chondrocytes. The similarity of the response of chondrocytes to indomethacin and diclofenac was striking. Both drugs showed no effect on proteoglycan secretion at concentrations up to and including $10 \mu \mathrm{g} / \mathrm{ml}$, and both strongly inhibited proteoglycan secretion at 50 and $100 \mu \mathrm{g} / \mathrm{ml}$. The pattern of response to different concentrations was established in the initial period of exposure to the drug and did not change for the duration of the experiment. Annefeld et al reported similar effects for these two drugs using a very different system based on morphometric assessment of chondrocyte ultrastructure. ${ }^{7}$ The morphometric evaluation of rat articular chondrocytes after 12 weeks of twice weekly administration of drugs at doses corresponding to the drugs' $E D_{50}^{5}$ showed similar scores for diclofenac and indomethacin for a number of variables, including endoplasmic reticulum, Golgi bodies, mitochondria, fat vacuoles, glycogen, and mortality. It was concluded that indomethacin and diclofenac both inhibited chondrocyte metabolic activity, whereas piroxicam and tiaprofenic acid studied in the same system had stimulatory effects on the cells. Using a similar ultrastructural study, Fassbender observed that treatment of rats with indomethacin resulted in damage to chondrocyte ultrastructure, 
whereas tiaprofenic acid caused only insignificant changes. ${ }^{8}$ In experiments measuring proteoglycan biosynthesis by chondrocytes or cartilage explants various effects of indomethacin have been reported. Proteoglycan synthesis by bovine chondrocytes was inhibited by indomethacin at a concentration of $10^{-5} \mathrm{~mol} / \mathrm{l}$ in a study undertaken by Mitrovic et $\mathrm{al}^{3}$ but was not inhibited by $1.4 \times 10^{-5} \mathrm{M}$ indomethacin according to a report by Klamfeldt. ${ }^{9}$ Palmoski and Brandt found that proteoglycan synthesis in canine cartilage explants was unaffected by indomethacin at concentrations up to $15 \mu \mathrm{g} / \mathrm{ml}\left(4 \cdot 2 \times 10^{-5} \mathrm{~mol} / \mathrm{l}\right){ }^{10}$ and, surprisingly, in a recent paper by Muir et al ${ }^{11}$ proteoglycan synthesis by canine cartilage explants exposed to $5 \times 10^{-7}$ and $10^{-6} \mathrm{M}$ indomethacin was reported to be significantly lower than that of controls, but at the higher concentrations of $5 \times 10^{-6}$ and $10^{-5} \mathrm{~mol} / \mathrm{l}$ this drug had no significant suppressive effect on biosynthesis. Much higher concentrations of indomethacin were used by McKenzie et al, who found that cartilage explants from human osteoarthritic femoral heads synthesised significantly less proteoglycans when exposed to $0.5 \mathrm{mM}$ and $1 \mathrm{mM}$, but not $0 \cdot 1 \mathrm{mM}(36 \mu \mathrm{g} / \mathrm{ml})$, indomethacin. ${ }^{12}$ The response of degenerate cartilage to indomethacin was also studied by Brandt and Palmoski, who reported that at $10^{-6} \mathrm{~mol} / \mathrm{l}$ this NSAID suppressed by $46 \%$ the glycosaminoglycan synthesis of atropic canine knee cartilage, whereas in normal cartilage proteoglycan synthesis was unaffected. ${ }^{13}$ Relatively few studies have described the in vitro effects of diclofenac on proteoglycan biosynthesis. Mohr and Kirkpatrick measured the incorporation of ${ }^{35} \mathrm{SO}_{4}^{--}$ into rabbit chondrocyte cultures and reported that the presence of $5 \mu \mathrm{g} / \mathrm{ml}$ of diclofenac had no effect. ${ }^{14}$

In all these studies proteoglycan synthesis was measured at a single timepoint only after a period of exposure to the drug. In addition, the different sources of cartilage, the variable periods of exposure to the drug, the widely varying range of concentrations selected for study, and other aspects of the experimental designs have all contributed to the confused and inconsistent results obtained in the above in vitro assessments of NSAIDs. From the present study we may conclude that although indomethacin and diclofenac showed an inhibitory effect on proteoglycan secretion at 50 and $100 \mu \mathrm{g} / \mathrm{ml}$, such concentrations would be in excess of those achieved in synovial fluid during normal therapeutic administration of these drugs to arthritic patients. ${ }^{15} 16$

The effects of tiaprofenic acid and ketoprofen on the secretion of proteoglycan by chondrocytes were essentially similar in our experiments. These two drugs are structurally related in that the benzene ring attached to the 2 position of propionic acid in ketoprofen is replaced by a thiophene ring in ? tiaprofenic acid. As the aromatic character of thiophenes is high it is perhaps not unexpected that these two drugs should behave similarly.

Tiaprofenic acid and, especially, ketoprofen hac comparatively weak inhibitory effects on proteo-त्ष glycan secretion. The effect was inconsistent and $P$ was not strongly dependent on concentration (sees Figs 5 and 6). Inhibition of proteoglycan secretion $\vec{b}$ by high doses of these drugs was most evident duringdays $2-4$, after which the percentage inhibition aw 50 and $100 \mu \mathrm{g} / \mathrm{ml}$ diminished with time. Tiaprofenico acid at $10 \mu \mathrm{g} / \mathrm{ml}$, a concentration which is achieved $\overline{\mathrm{p}}$ clinically in serum and synovial fluid, ${ }^{17}$ significantly depressed proteoglycan secretion during the initiaij culture interval, but the effect was very slight and was transient. Studies by other workers have $\mathrm{O}^{N}$ assessed the in vitro effects of tiaprofenic acid on? murine patella cartilage ${ }^{18}$ canine articular cartilage $\vec{z}_{\vec{r}}$ explants, ${ }^{19}$ and chick ${ }^{20}$ and rabbit ${ }^{16}$ chondrocytew cultures. In none of these studies was a significant inhibition of proteoglycan synthesis reported, but these investigations did not include concentrations ${ }^{\circ}$ of tiaprofenic acid as high as 50 and $100 \mu \mathrm{g} / \mathrm{ml}, 0$ which were found to be inhibitory in the presents study. de Vries et al reported that tiaprofenic acid at $30 \mu \mathrm{g} / \mathrm{ml}$ did not suppress glycosaminoglycan syn thesis by murine patella cartilage in vitro, ${ }^{18}$ but the explanted patellae were exposed to the drug for only 30 minutes, so the acute response to the drugg measured by these authors could not be compared with our study, which may be considered as equi-? valent to a chronic effect.

The two sulphated polysaccharides studied, glycosê를 aminoglycan polysulphate ester and sodium pento san polysulphate, represent a relatively new class of antiarthritic agents, whose pharmacological proper- 0 요 ties have been recently reviewed. ${ }^{121}$ Their effects 3 on chondrocyte proteoglycan secretion were, cor-윽 respondingly, quite different from those of the $>$ NSAIDs. During the initial period of exposure (days 0-2) proteoglycan secretion was significantly sup- ̃N pressed by concentrations of 50 and $100 \mu \mathrm{g} / \mathrm{ml}$ of both drugs. Although this pattern was maintained for sodium pentosan polysulphate, glycosamino- $-\tilde{\omega}$ glycan polysulphate ester was not inhibitory ato any concentration during the subsequent cultureo intervals. The significant and substantial stimulation of proteoglycan secretion in the presence of low: concentrations of both these sulphated polysac- -0 charides, once established, persisted for the duration? of the experiment. This result is in contrast with the effects of the NSAIDs, none of which caused any묘 significant stimulation of proteoglycan secretiono over the concentration range examined. Our findings that sulphated polysaccharides could stimulate pro응 
teoglycan secretion are consistent with other reports where chondrocyte cultures were employed. 2223 The stimulation of proteoglycan secretion by these drugs in this study occurred at concentrations which could be readily achieved in cartilage during human administration of drugs to patients for the treatment of osteoarthritis. ${ }^{24}$ Our in vitro findings are therefore consistent also with the chondroprotective action of these drugs reported to occur in a number of animal models. ${ }^{121}$

The simple method used in the present study appears to offer distinct advantages over existing procedures. By reducing the tedium and experimental variability associated with other methods it has facilitated the evaluation of the effects of drugs on chondrocyte proteoglycan biosynthesis under a variety of conditions. This method should therefore find application not only in the screening of antiarthritic drugs but also in other areas where knowledge of proteoglycan biosynthesis in cell or organ cultures is an experimental objective.

We gratefully acknowledge the competent assistance of Mrs Anne Darvodelsky in undertaking some of the proteoglycan assays and Ms Lynne Campbell for typing the manuscript. We also thank $\mathrm{Mr}$ John Andrews of these laboratories for his useful suggestions during the development of the assay procedure and the National Health and Medical Research Council for supporting this work.

\section{References}

1 Burkhardt D, Ghosh P. Laboratory evaluation of antiarthritic drugs as potential chondroprotective agents. Semin Arthritis Rheum 1987; 17 (suppl 1): 3-34.

2 Mason R M, Lineham J D, Phillipson M A, Black C M. Selective inhibition of proteoglycan and hyaluronate synthesis in chondrocyte cultures by cyclofenil diphenol, a non-steroidal weak oestrogen. Biochem J 1984; 223: 401-12.

3 Mitrovic D, McCall E, Front P, April F, Darmon N, Dray F. Anti-inflammatory drugs, prostanoid and proteoglycan production by cultured bovine articular chondrocytes. Prostaglandins 1984; $28:$ : 417-34.

4 Schwartz N B, Dorfinan A. Stimulation of chondroitin sulphate proteoglycan production by chondrocytes in monolayer. Connect Tissue Res 1975; 3: 115-22.

5 Blumenkrantz N, Asboe-Hansen G. New method for quantitative determination of uronic acids. Anal Biochem 1973; 54: 484-9.

6 Sokal R R, Rohlf F J. Biometry. San Francisco: W H Freeman, 1969: $239-46$.

7 Annefeld M, Raiss R, Cleres C. Einfluss steroidaler und nichtsteroidaler Antiphlogistika auf die Ultrastruktur von Chondrozyten der Ratte. Elektronenoptische und morphometrische Studie. Arzneimittelforschung 1984; 34: 1763-5.

8 Fassbender H G. Role of chondrocytes in the development of osteoarthritis. Am J Med 1987; 83 (suppl 5A): 17-24.
9 Klamfeldt A. Effect of indomethacin and hydrocortisone upon joint tissue in vitro. Scand J Rheumatol 1985; 14: 225-9.

10 Palmoski M J, Brandt K D. Effects of some nonsteroidal antiinflammatory drugs on proteoglycan metabolism and organisation in canine articular cartilage. Arthritis Rheum 1980; 23: 1010-20.

11 Muir H, Carney S L, Hall L G. Effects of tiaprofenic acid and other NSAIDs on proteoglycan metabolism in articular cartilage explants. Drugs 1988; 35 (suppl 1): 15-23.

12 McKenzie L S, Horsburgh B A, Ghosh P, Taylor T K F. Effect of anti-inflammatory drugs on sulphated glycosaminoglycan synthesis in aged human articular cartilage. Ann Rheum Dis 1976; 35: 487-97.

13 Brandt K D, Palmoski M J. Proteoglycan content determines the susceptibility of articular cartilage to salicylate-induced suppression of proteoglycan synthesis. J Rheumatol 1983; (suppl 9): 78-80.

14 Mohr W, Kirkpatrick C J. In vitro experiments with chondrocytes. In: Munthe E, Bjelle A, eds. Effect of drugs on osteoarthrosis. Berne: Hans Huber, 1984: 75-89.

15 Flouvat B. Pharmacologic properties and pharmacokinetic pattern of piroxicam, a new anti-inflammatory agent. Proceedings of the 15th International Congress of Rheumatology. Paris: International Congress of Rheumatology, 1981: 1-15.

16 Shinmei M, Shimada K, Shigeno Y, Kikuchi K, Miyazaki K, Shimomura $Y$. Effects of anti-inflammatory drugs on proteoglycan synthesis and degradation in rabbit articular chondrocytes in vitro. In: Nilsen $\mathrm{O}$ G, ed. New trends in rheumatology. Vol 3. Geneva: Excerpta Medica, 1985: 59-68.

17 Daymond T J, Herbert R. Simultaneous bioavailability of tiaprofenic acid (Surgam) in serum and synovial fluid in patients with rheumatoid arthritis. Rheumatology 1982; 7: 188-93.

18 de Vries B J, van den Berg W B, Vitters E, et al. Effects of NSAIDs on the metabolism of sulphated glycosaminoglycans in healthy and (post) arthritic murine articular cartilage. Drugs 1988, 35 (suppl 1): 24-32.

19 Carney S L, Muir H, Hall L G. A study of the effects of nonsteroidal anti-inflammatory drugs on proteoglycan metabolism in articular cartilage explants. Proceedings of the 11th Congress of the European League Against Rheumatism. Athens: European League Against Rheumatism, 1987.

20 Kajiwara T, Mitsui K, Fujii K, Murota K. Effects of NSAIDs and synovial fluid on proteoglycan metabolism. Proceedings of the International Symposium on Rheumatology. Tokyo: International Symposium on Rheumatology, 1987.

21 Burkhardt D, Ghosh P. Laboratory evaluation of glycosaminoglycan polysulphate ester for chondroprotective activity: a review. Curr Ther Res 1986; 40: 1034-53.

22 Adam M, Krabcová M, Musilová J, Pesáková V, Brettschneider I, Deyl Z. Contribution to the mode of action of glycosaminoglycan-polysulphate (GAGPs) upon human osteoarthritic cartilage. Biochemical study of the collagen and proteoglycan turnover. Arzneimittelforschung 1980; 30: 1730-2.

23 Verbruggen G, Veys E M. Proteoglycan metabolism of connective tissue cells. An in vivo technique and its relevance to in vivo conditions. In: Verbruggen G, Veys E M, eds. Degenerative joints, test tubes, tissues, models, man. Amsterdam: Excerpta Medica, 1982: 113-26.

24 Muller W, Panse P, Brand S, Staubli A. In vivo study of the distribution, affinity to cartilage and metabolism of glycosaminoglycan polysulphate (GAGPs, Arteparon). Z Rheumatol 1983; 42: 355-61. 